\title{
Mediastinal synovial sarcoma: A case report and literature review
}

\author{
Linda SL Cheng MBChB ${ }^{1}$, Gary MK Tse MBBS FRCPC ${ }^{2}$, Wilson WL Li ${ }^{1}$, \\ TW Lee FRCS ${ }^{1}$, Anthony PC Yim DM FRCS ${ }^{1}$
}

LSL Cheng, GMK Tse, WWL Li, TW Lee, APC Yim. Mediastinal synovial sarcoma: A case report and literature review. Can Respir J 2003;10(7):393-395.

Synovial sarcomas are uncommon soft tissue tumours. Immunohistochemistry and cytogenetic techniques are essential for proper diagnosis and differentiation from other spindle cell neoplasms. A case of mediastinal synovial sarcoma is described, of which the unusual location, diagnosis and treatment form the basis of this report.

\section{Un synovialome médiastinal : Rapport de cas et analyse bibliographique}

\begin{abstract}
Les synovialomes sont des tumeurs peu courantes des tissus mous. L'immunohistochimie et les techniques cytogénétiques sont essentielles pour poser un diagnostic convenable et obtenir une différenciation des autres néoplasmes à cellules fusiformes. Un cas de synovialome médiastinal est décrit, dont l'emplacement inhabituel, le diagnostic et le traitement forment le fondement du présent rapport.
\end{abstract}

Key Words: Mediastinum; Synovial sarcoma

Synovial sarcomas are uncommon but well-defined soft tis5 sue tumours, accounting for $5 \%$ to $10 \%$ of all soft tissue sarcomas (1). It occurs mainly in adolescents and young adults, usually arising in the extremities and the area of large joints. Intrathoracic involvement is unusual, with most known cases occurring as metastases from distant organs. We report the case of a primary synovial sarcoma in the mediastinum, which is a rare site for the tumour to arise.

\section{CASE PRESENTATION}

A 60-year-old woman was referred with an incidentally discovered abnormal shadow in the right mediastinum on routine chest radiography (Figure 1). The patient was a nonsmoker with a past medical history of pulmonary tuberculosis. She had an occasional cough without sputum production, but no other respiratory symptoms. Physical examination was unremarkable. Results of routine laboratory studies were normal. Contrast-enhanced computed tomography $(\mathrm{CT})$ of the thorax showed a large paraspinal mass measuring $6.8 \mathrm{~cm} \times 5.9 \mathrm{~cm} \times 5 \mathrm{~cm}$ in size (Figure 2). The mass was located in the right middle mediastinum, next to the apical segment of the right lower lobe. The lesion had a lobular margin with some calcified foci in the peripheral area. No significant mediastinal lymphadenopathy was detected. No abnormalities were seen in the left side. Positron emission

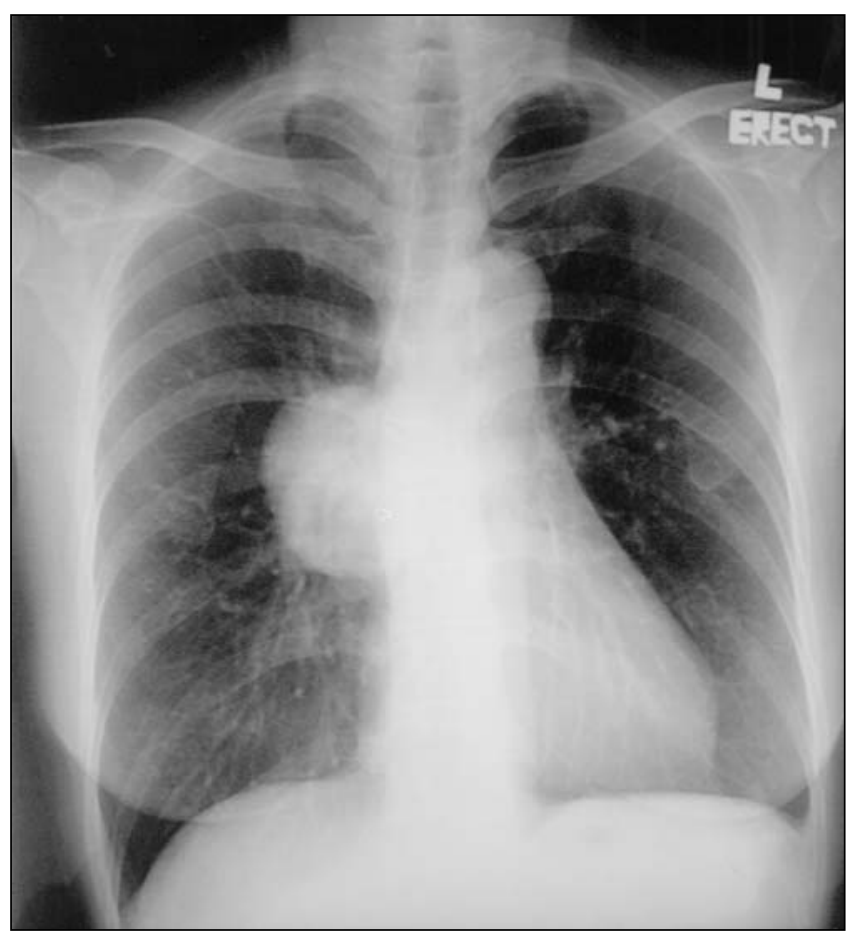

Figure 1) Chest radiography demonstrating a right middle mediastinal mass

${ }^{1}$ Division of Cardiothoracic Surgery, Department of Surgery; ${ }^{2}$ Department of Anatomical and Cellular Pathology, The Chinese University of Hong Kong, Prince of Wales Hospital, Hong Kong Special Administrative Region, China

Correspondence: Dr Anthony PC Yim, Division of Cardiothoracic Surgery, Department of Surgery, The Chinese University of

Hong Kong, Prince of Wales Hospital, Shatin, NT, Hong Kong Special Administrative Region, China. Telephone +852-2632-2629, fax +852-

2647-8273,e-mail yimap@cuhk.edu.hk 


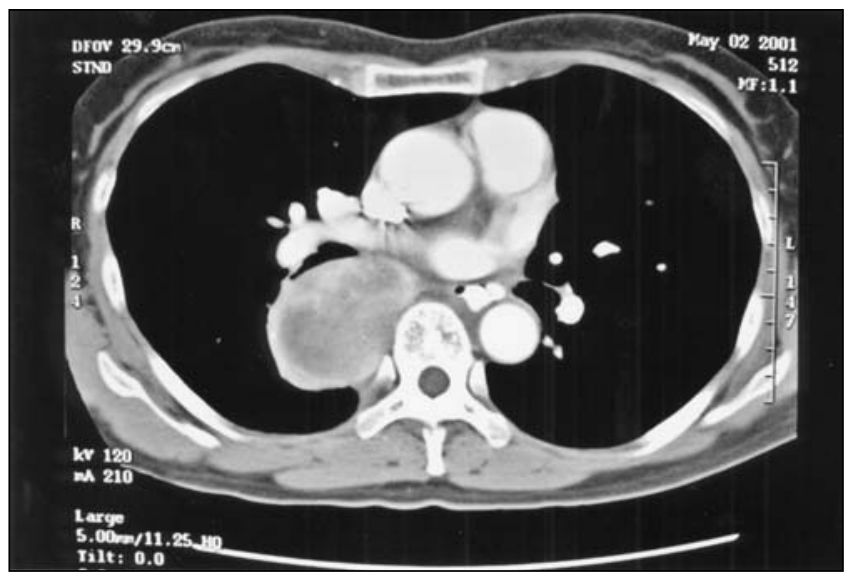

Figure 2) Computed tomography of the chest showing a large right paraspinal mass, next to the apical segment of the right lower lobe

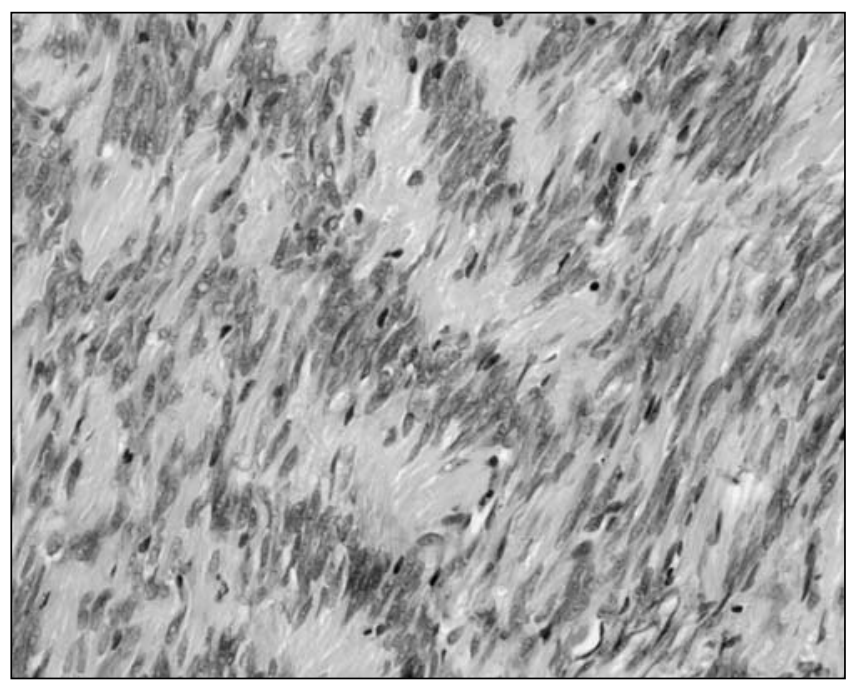

Figure 3) Photomicrograph showing prominent palisading pattern on the tumour, with cell nuclei arranged in parallel bands. (Hematoxylin and eosin stain, original magnification $\times 200$ )

tomography (PET) scan showed activity of the lesion without signs of distant metastasis. Magnetic resonance imaging (MRI) of the thorax excluded invasion of the lesion into the spinal canal. CT-guided biopsy showed a spindle cell tumour, with the differential diagnosis of schwannoma or solitary fibrous tumour. The patient was then scheduled for videoassisted thoracic surgery (VATS) for resection of this lesion.

Intraoperatively, through a right thoracotomy at the fifth intercostal space, a large paraspinal mass was found, with the right lung draped over the tumour. The tumour was resected without difficulty. The specimen consisted of multiple friable pieces of soft tan/white tissue measuring in aggregate $7 \mathrm{~cm} \times$ $7 \mathrm{~cm} \times 4 \mathrm{~cm}$. Pathological examination of the resected specimen showed a cellular spindle cell neoplasm with prominent palisading pattern (Figure 3) and clear resection margins. The spindle cells showed a mild degree of nuclear pleomorphism and hyperchromasia (Figure 4). Mitotic activity was low, with a mitotic count of three to four per 10 high power fields. Within the tumour, occasional myxoid stromal areas, calcification and necrosis were seen. No definite epithelial component was

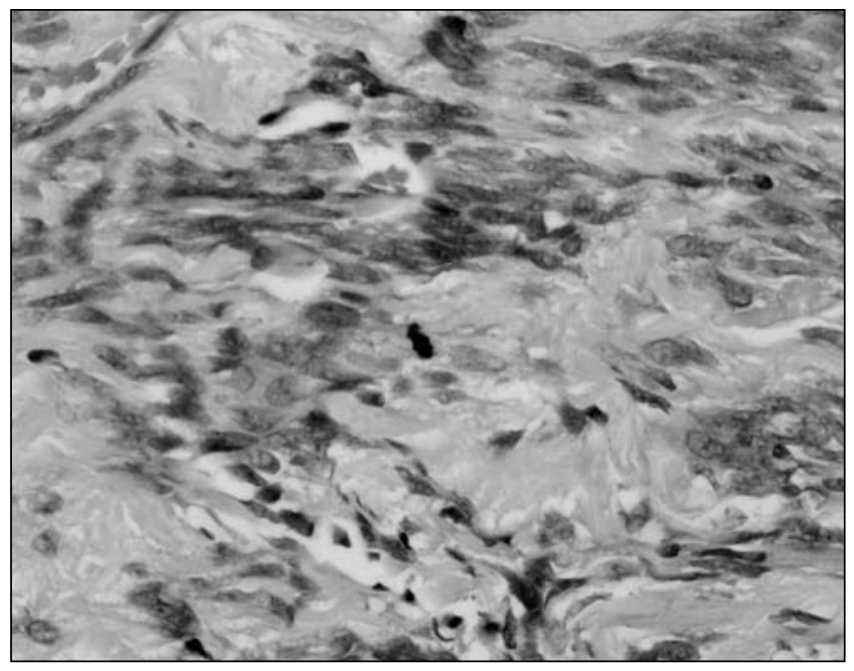

Figure 4) Photomicrograph showing some nuclear pleomorphism; a mitotic figure is seen in the centre. (Hemotoxylin and eosin stain, original magnification $\times 400$ )

identified. Immunohistochemical examination showed strong staining with vimentin (Dako, dilution 1:100), and weak and focal staining with cytokeratin (Cytokeratins-AE1/AE3, Dako, USA [dilution 1:300]; epithelial membrane antigen, Dako, USA [dilution 1:50]). Staining with S100 (Dako, USA [dilution 1:150]), desmin (Dako, USA [dilution 1:150]), CD34 (Novacastra, United Kingdom [dilution 1:30]) and actin (Dako, USA [dilution 1:1]) were negative. A diagnosis of a monophasic fibrous synovial sarcoma was made.

The postoperative course was uneventful, and the patient was discharged on postoperative day 3 . She was offered adjuvant radiation therapy based on the size of the tumour. However, this was declined by the patient. She continues to do well without evidence of recurrence after a follow-up period of three months.

\section{DISCUSSION}

Synovial sarcoma derives its name from its histomorphological resemblance to synovium. However, this is a misnomer, because more recent evidence suggests that the tumour is derived from primitive pluripotential mesenchyme, which is capable of synovial differentiation. This could explain its occurrence in unusual sites such as the lung (2), mediastinum $(3,4)$ and pleural cavity $(5-7)$. Histologically, synovial sarcomas are composed of a spindle cell and an epithelial cell element. The classic histological pattern is that of a biphasic tumour, composed of varying proportions of both elements. If the epithelial element is lacking, the tumour is referred to as a monophasic fibrous synovial sarcoma. Other subtypes are very uncommon, including the monophasic epithelial synovial sarcoma and the undifferentiated type.

Intrathoracic synovial sarcomas are clinically often a diagnostic challenge due to their nonspecific presentation (2-7) Common symptoms include chest pain, shortness of breath, hemoptysis and cough. However, most patients present with a slow growing, painless mass. Radiographic examination should include chest radiography, CT and MRI of the tumour. Furthermore, as intrathoracic involvement by synovial sarcomas is more commonly due to metastasis, PET scans can be of 
value to detect concomitant lesions in other sites and assess the primary nature of the tumour. In addition, PET scans can be helpful in estimating tumour grade $(8,9)$ and evaluating response to neoadjuvant chemotherapy (9).

Differential diagnosis for synovial sarcomas should include other spindle cell tumours such as malignant peripheral nerve sheath tumours, soft tissue c, spindle cell carcinomas, spindle cell thyomas, mesotheliomas and solitary fibrous tumours of the pleura. In this case, the high cellularity of the lesion excluded a solitary fibrous tumour. For differentiation from the other lesions, detection of antigenic profiles by immunohistochemistry can be essential. Expression of CD34 is expected for a solitary fibrous tumour but not for a synovial sarcoma, and expression of S100 protein indicates a peripheral nerve sheath tumour in this setting. For tumours with smooth muscle differentiation, like leiomyosarcomas and leiomyomas, immunostains are reactive toward the intermediate filaments like desmin or actin. Furthermore, strong coexpression of the intermediate filament vimentin indicates mesenchymal differentiation, while weak coexpression of cytokeratin suggests epithelial differentiation. This is characteristic for a group of uncommon sarcomas including notably synovial sarcomas, epithelioid sarcomas and rhabdoid tumours. In this case, the absence of the epithelial component supported a diagnosis of a monophasic synovial sarcoma.

In addition to immunohistochemical studies, cytogenetic techniques are helpful in confirming the diagnosis. Synovial sarcomas are known to have a characteristic chromosomal translocation between chromosomes $\mathrm{X}$ and $18(7,10)$. This specific diagnostic tool can sometimes be necessary to establish the diagnosis of a synovial sarcoma. Due to the characteristic features of the tumour, cytogenetic techniques were not used in this case.

\section{REFERENCES}

1. Weiss SW, Goldblum JR. Synovial sarcoma. In: Weiss SW, Goldblum JR, eds. Soft Tissue Tumors. St. Louis: Mosby-Year Book, 2001:1483-509.

2. Zeren H, Moran CA, Suster S, Fishback NF, Koss MN. Primary pulmonary sarcomas with features of monophasic synovial sarcoma: A clinicopathological, immunohistochemical, and ultrastructural study of 25 cases. Hum Pathol 1995;26:474-80.

3. Witkin GB, Miettinen M, Rosai J. A biphasic tumor of the mediastinum with features of synovial sarcoma. A report of four cases. Am J Surg Pathol 1989;13:490-9.

4. Le Marc'hadour F, Pasquier B, Leroux D, Jacrot M. Mediastinal synovial sarcoma with $\mathrm{t}(\mathrm{X} ; 18)$. Cancer Genet Cytogenet 1991;55:265-7.

5. Cappello F, Barnes L. Synovial sarcoma and malignant mesothelioma of the pleura: Review, differential diagnosis and possible role of apoptosis. Pathology 2001;33:142-8.

6. Aubry MC, Bridge JA, Wickert R, Tazelaar HD. Primary monophasic synovial sarcoma of the pleura: Five cases confirmed by the presence of SYT-SSX fusion transcript. Am J Surg Pathol 2001;25:776-81.

7. Essary LR, Vargas SO, Fletcher CD. Primary pleuropulmonary
Traditionally, synovial sarcomas carry a poor prognosis. However, with careful staging and more sophisticated diagnostic techniques, survival rates have improved. Overall, five-year survival rates of up to $88 \%$ have been reported $(11,12)$. Furthermore, it is now clear that synovial sarcoma patients can be divided into low and high risk groups, with significant effect on survival. Good prognostic factors for survival of a primary synovial sarcoma include small tumour size (smaller than 5 $\mathrm{cm}$ ), clear margin of resection, low mean mitotic activity (less than 15 mitoses per 10 high power fields), peripheral location of the tumour, absence of necrosis in the tumour and young patient age (younger than 25 years) $(10,11)$.

The main treatment modality for primary synovial sarcomas continues to be radical local resection $(11,12)$. Although VATS resection of mediastinal tumours is certainly feasible, we do not recommend VATS for tumours larger than $4 \mathrm{~cm}$, not primarily because of technical difficulties, but because ribs have to be excessively spread to retrieve the specimen. This tends to negate the benefit of minimal access surgery. Nevertheless, we do recommend the routine use of VATS exploration in all surgical cases of pulmonary or mediastinal malignancy to exclude any contraindications for resection (13). Whether adjuvant chemotherapy or radiotherapy contributes to better local control or survival rates remains controversial at present $(11,12)$.

\section{CONCLUSIONS}

Synovials sarcoma arising from the mediastinum are distinctively rare. To the best of our knowledge, only five such cases have been reported in the English literature $(3,4)$. We report a sixth case of primary mediastinal synovial sarcoma and the first monophasic case.

synovial sarcoma: Reappraisal of a recently described anatomic subset. Cancer 2002;94:459-69.

8. Schwarzbach MH, Dimitrakopoulou-Strauss A, Willeke F, et al. Clinical value of [18-F]] fluorodeoxyglucose positron emission tomography imaging in soft tissue sarcomas. Ann Surg 2000;231:380-6.

9. Folpe AL, Lyles RH, Sprouse JT, Conrad EU 3rd, Eary JF. (F-18) fluorodeoxyglucose positron emission tomography as a predictor of pathologic grade and other prognostic variables in bone and soft tissue sarcoma. Clin Cancer Res 2000;6:1279-87.

10. Kawai A, Woodruff J, Healey JH, Brennan MF, Antonescu CR, Ladanyi M. SYT-SSX gene fusion as a determinant of morphology and prognosis in synovial sarcoma. N Engl J Med 1998;338:153-60.

11. Bergh P, Meis-Kindblom JM, Gherlinzoni F, et al. Synovial sarcoma: Identification of low and high risk groups. Cancer 1999;85:2596-607.

12. Trassard M, Le Doussal V, Hacene K, et al. Prognostic factors in localized primary synovial sarcoma: A multicenter study of 128 adult patients. J Clin Oncol 2001;19:525-34.

13. Yim AP. VATS major pulmonary resection revisited-controversies, techniques, and results. Ann Thorac Surg 2002;74:615-23. 


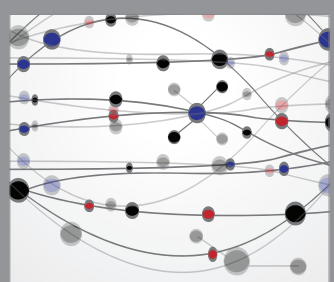

The Scientific World Journal
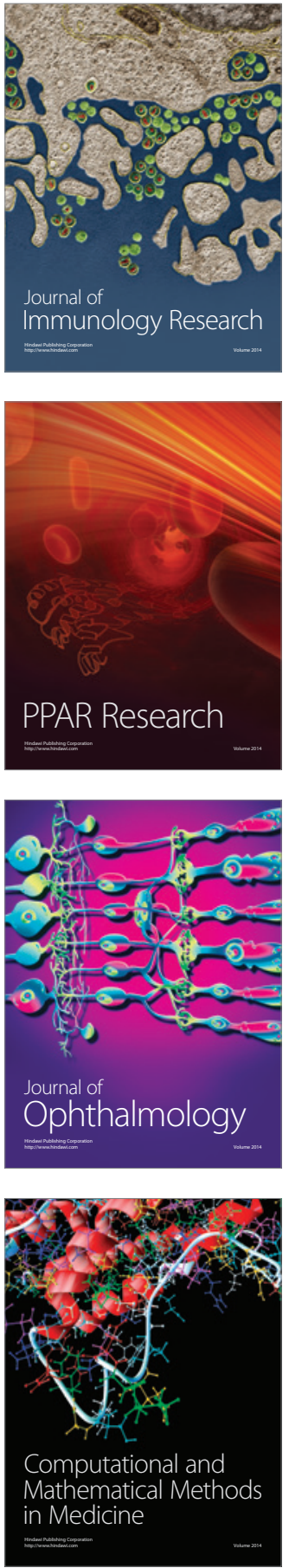

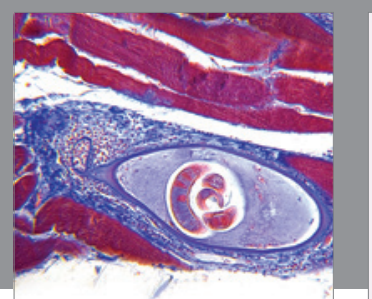

Gastroenterology Research and Practice

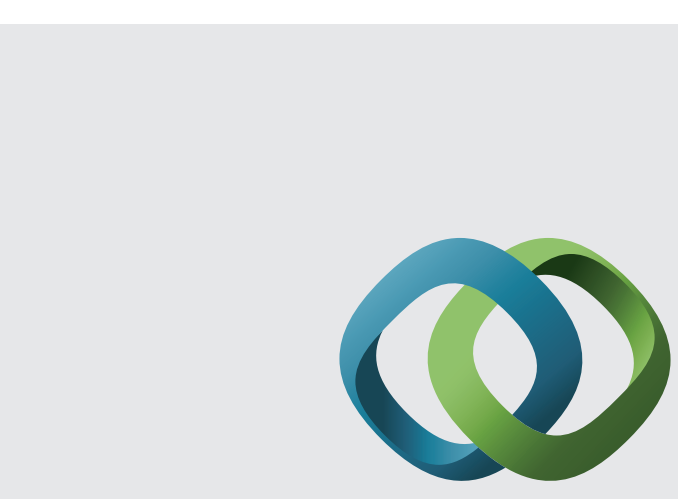

\section{Hindawi}

Submit your manuscripts at

http://www.hindawi.com
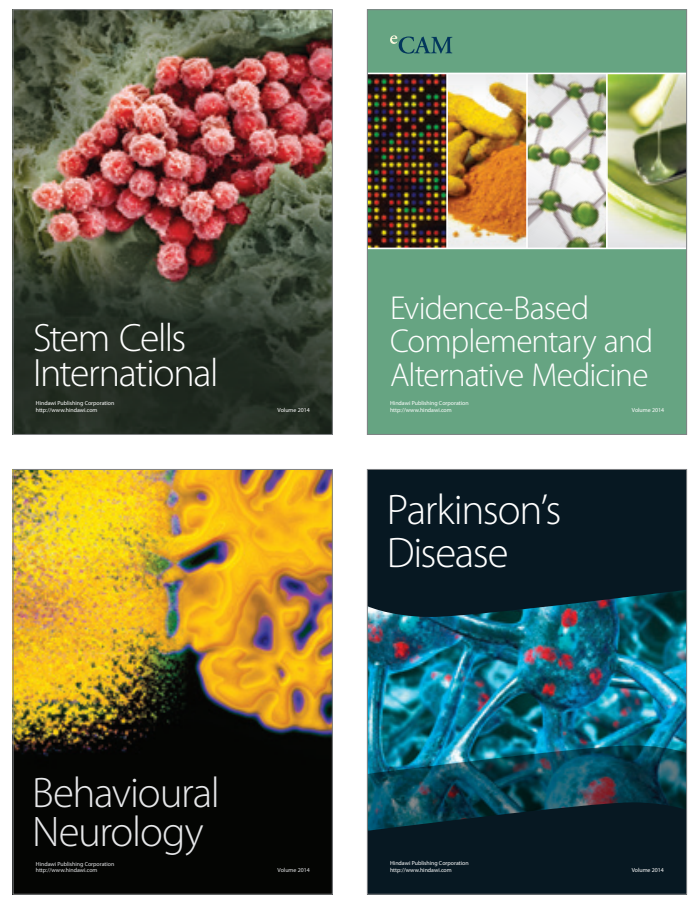
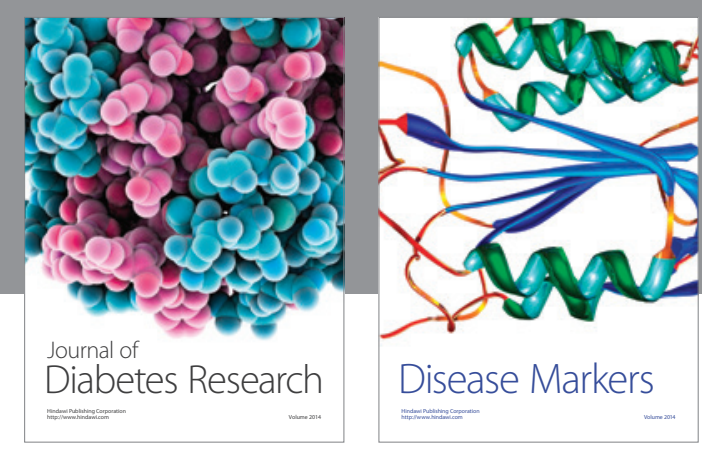

Disease Markers
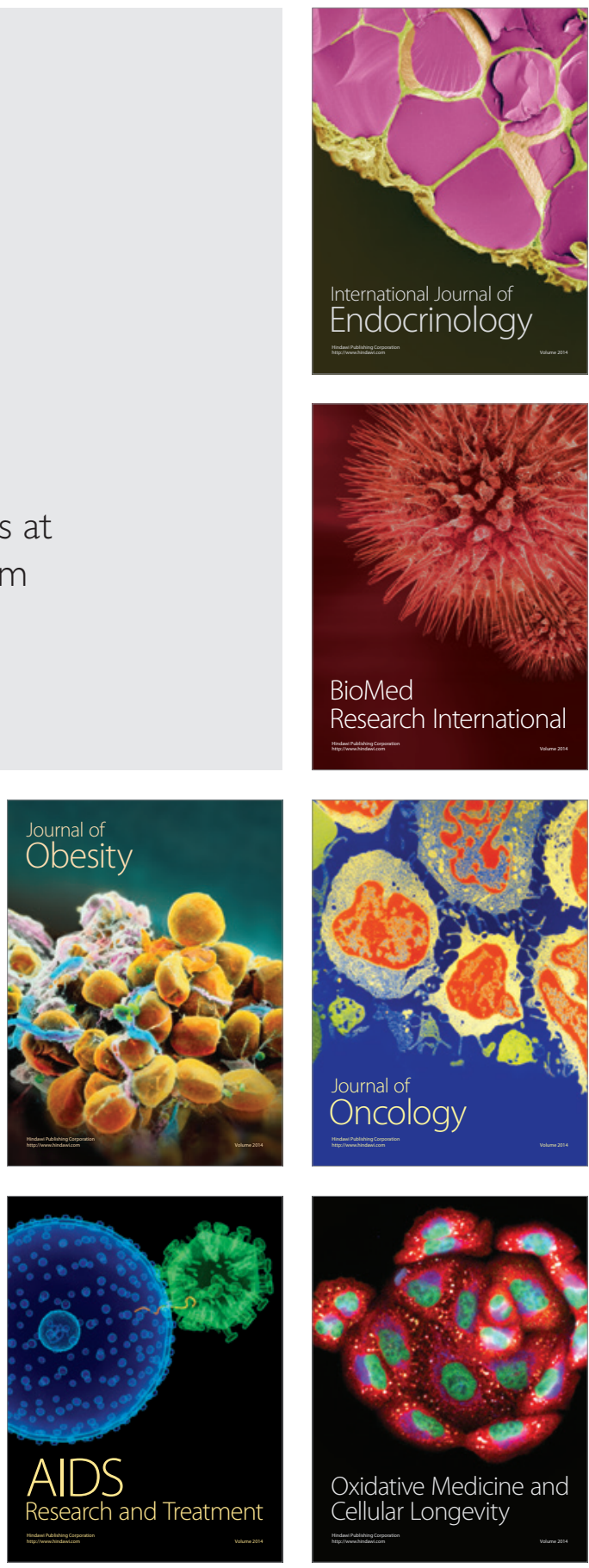